\title{
Characterization of the adipokinetic hormone receptor of the anautogenous flesh fly, Sarcophaga crassipalpis
}

\author{
Magdalena Bil a ${ }^{\text {, Iris Timmermans }}{ }^{\mathrm{a}}$, Heleen Verlinden ${ }^{\mathrm{b}, 1}$, Roger Huybrechts ${ }^{\mathrm{a}, *, 1}$ \\ ${ }^{a}$ Research Group of Insect Physiology and Molecular Ethology, KU Leuven, Naamsestraat 59, B-3000, Belgium \\ ${ }^{\mathrm{b}}$ Research Group of Molecular Developmental Physiology and Signal Transduction, KU Leuven, Naamsestraat 59, B-3000, Belgium
}

\section{A R T I C L E I N F O}

\section{Article history:}

Received 5 February 2016

Received in revised form 31 March 2016

Accepted 6 April 2016

Available online 7 April 2016

\section{Keywords:}

Adipokinetic hormone

Adipokinetic hormone receptor

$\mathrm{G}$ protein-coupled receptor

Differential expression

Flesh fly

Anautogeny

\begin{abstract}
A B S T R A C T
Adipokinetic hormone $(\mathrm{AKH})$ is an insect neuropeptide mainly involved in fat body energy mobilization. In flies (Phormia regina, Sarcophaga crassipalpis), bugs (Pyrrhocoris apterus) and cockroaches (Periplaneta americana) AKH was also demonstrated to be involved in the regulation of digestion. This makes AKH an important peptide for anautogenous female flies that need to feed on a supplementary protein meal to initiate vitellogenesis, the large scale synthesis of yolk proteins and their uptake by the developing oocytes.

Flesh fly AKH, originally identified as Phormia terraenovae hypertrehalosemic hormone (PhoteHrTH), functions through activation of the AKH receptor (AKHR). This is a G protein-coupled receptor that is the orthologue of the human gonadotropin-releasing hormone receptor. Pharmacological characterization indicated that the receptor can be activated by two related dipteran $\mathrm{AKH}$ ligands with an $\mathrm{EC}_{50}$ value in the low nanomolar range, whereas micromolar concentrations of the Tribolium castaneum AKH were needed.

Consistent with the energy mobilizing function of AKH, the receptor transcript levels were most abundant in the fat body tissue. Nonetheless, Sarcophaga crassipalpis AKHR transcript levels were also high in the brain, the foregut and the hindgut. Interestingly, the receptor transcript numbers were reduced in almost all measured tissues after protein feeding. These changes may enforce the use of ingested energy carrying molecules prior to stored energy mobilization.
\end{abstract}

(ㄷ) 2016 Elsevier Ltd. All rights reserved.

\section{Introduction}

Adult females of anautogenous insects require a supplementary protein meal as a nutrient source for egg maturation. An important event in insect egg development is vitellogenesis, the large scale production of yolk proteins (YPs) in the fat body and their subsequent uptake by the growing oocytes. The YP precursor gene expression is tightly controlled by the nutritional state of the organism. It remains arrested at the previtellogenic level until activated by particular signaling pathways after the protein meal (Attardo et al., 2003). For this reason, the control of feeding and digestion processes is crucial for the anautogenous reproductive strategy. One of the neuropeptides involved in the midgut enzymatic activity regulation of the flesh fly Sarcophaga crassipalpis is adipokinetic hormone (AKH) (Bil et al., 2014).

\footnotetext{
* Corresponding author.

E-mail address: Roger.Huybrechts@bio.kuleuven.be (R. Huybrechts).

${ }^{1}$ Equally contributing senior authors.
}

AKH was first isolated from the migratory locust, Locusta migratoria (Stone et al., 1976) and is one of the best studied insect neuropeptides. It is a member of a large group of structurally related peptides, the $\mathrm{AKH} /$ red pigment-concentrating hormone $(\mathrm{RPCH})$ family. AKH is one of the most abundant neuropeptides present in most insects and usually consists of 8-10 amino acids. AKHs share some common structural features of pyroglutamic acid at the N-terminal end, aromatic amino acids at the fourth position (in the majority of cases, phenylalanine), a tryptophan at the eighth position and an amide at the C-terminal end (Marco et al., 2011; Gäde, 2009). It is mainly involved in the mobilization of stored energy carrying molecules to support all high energetic processes, as for example flying (Van der Horst, 2003). But AKH has a pleiotropic nature and also acts directly on flight muscles influencing the flight speed (Goldsworthy et al., 1979), effects walking activity in non-flying insects (Kodrik et al., 2000), stimulates the frequency of the heart beating (Scarborough et al., 1984), participates in the immune responses (Goldsworthy et al., 2005) and in the stress reactions (Kodrik, 2008; Kodrik et al., 2005), is involved in egg development (Abdel-Latief and Hoffmann, 2007) and even 
regulates digestive activity (Kodrik et al., 2012; Bil et al., 2014; Bodláková et al., 2016).

The physiological functions of AKH are mediated by AKH receptors (AKHRs), rhodopsin-like $G$ protein-coupled receptors (GPCRs). AKHR was first identified and pharmacologically characterized in the fruit fly, Drosophila melanogaster, and in the silkworm, Bombyx mori (Staubli et al., 2002). Upon ligand binding, AKHR has been demonstrated to couple through $\mathrm{G}_{\mathrm{q}}$ protein, resulting in an increase of intracellular $\mathrm{Ca}^{2+}$ (Caers et al., 2016), and through $\mathrm{G}_{\mathrm{s}}$, causing increased intracellular cAMP levels (Shi et al., 2011).

In this study, we have identified and cloned the SarcrAKHR. We used Chinese hamster ovary ( $\mathrm{CHO}$ ) cells stably expressing the promiscuous $G_{\alpha 16}$ protein (causing an increase in intracellular $\mathrm{Ca}^{2+}$ levels upon activation) for pharmacological characterization. In addition, we investigated the receptor transcript levels under different nutritional regimes in adult female flies.

\section{Materials and methods}

\subsection{Insect rearing}

Anautogenous flesh flies, Sarcophaga crassipalpis, were kindly provided by the laboratory breading of Professor D. A. Hahn (Department of Entomology and Nematology, The University of Florida). Flies were bred under the specific laboratory conditions of $24^{\circ} \mathrm{C}$ and a light:dark cycle of $14: 10 \mathrm{~h}$. Flies in the cages selected for the experiments were kept under sugar and water diet only, to ensure no earlier increase of digestive activity and subsequent development of their ovaries. The other flies that were not used for any experiments were fed with additional pieces of cow liver to continue normal egg development and larviposition to preserve the culture.

\subsection{Adipokinetic hormone immunohistochemistry staining}

Immunocytochemistry staining was performed on corpora cardiaca (CC) dissected from four days old sugar fed female flies. Subsequently the tissues were fixated in $0.1 \mathrm{M}$ phosphate-buffered saline (PBS) solution containing $2 \%$ paraformaldehyde for $4 \mathrm{~h}$. Afterwards CCs were rinsed with PBS and incubated in the blocking buffer (100 ml $0.15 \mathrm{M}$ PBS; $3 \mathrm{~g}$ bovine serum albumin (BSA); $2 \mathrm{ml}$ Triton X-100)at room temperature. Afterwards the tissues were covered with the primary antibodies diluted in the blocking buffer containing 1\% Triton X-100 in dilution 1:4000 and incubated overnight at $4{ }^{\circ} \mathrm{C}$. The primary rabbit antibodies (polyclonal) used in this study were originally prepared against $D$. melanogaster $\mathrm{AKH}$ (Isabel et al., 2005; Veenstra, pers. commun.), which has an identical amino acid sequence compared to SarcrAKH. The following day the CCs were divided ad random into two groups. With two different methods, the localization of the primary antibodies was visualized. The first group was rinsed with Tris buffer and incubated in the secondary antibodies GAR-biotin (goat anti rabbit secondary antibodies, Dako) diluted in Tris buffer in dilution 1:200. Afterwards they were rinsed once again with Tris buffer and incubated with streptavidin-horseradish peroxidase (Dako). After the incubation the preparations were again rinsed with Tris and $0.1 \mathrm{M}$ sodium acetate buffers. Subsequently they were colored with the Shu coloring solution ( $5 \mathrm{~g}$ nickel ammonium sulfate, $80 \mathrm{mg}$ ammonium chloride, $400 \mathrm{mg} \beta$-D-glucose, $2 \mathrm{mg}$ glucose oxidase, $100 \mathrm{mg}$ diaminobenzidine tetrahydrochloride), washed and colored with eosin for tissue visualization and embedded in paraffin. The other group was rinsed with PBS buffer and incubated with the secondary fluorescent labeled antibodies GAR/FITC (fluorescein isothiocyanate) diluted in PBS containing 3\% bovine serum albumin and $1 \%$ Triton X-100 in dilution 1:2000. Afterwards the preparations were rinsed with PBS and subsequently covered with mowiol mounting medium (Sigma-Aldrich) and transparent nail polish.

\subsection{RNA isolation and CDNA synthesis}

Sixty sugar-fed female flies were used for the experiment and subsequently divided ad random into two groups. The first group of insects remained under sugar and water diet, whilst the second group was offered a piece of cow liver for $45 \mathrm{~min}$. Five hours after the beginning of this 45 min feeding time, seven different tissues including brain, foregut, midgut, hindgut, fat body, ovaries and Malpighian tubules were dissected with great care in Sarcophaga Ringer's solution containing $121.5 \mathrm{mM} \mathrm{NaCl} ; 10 \mathrm{mM} \mathrm{KCl} ; 1 \mathrm{mM}$ $\mathrm{NaH}_{2} \mathrm{PO}_{4} ; 10 \mathrm{mM} \mathrm{NaHCO} 3 ; 0.7 \mathrm{mM} \mathrm{MgCl} ; 2.2 \mathrm{mM} \mathrm{CaCl}$; pH 6.8 (Meulemans and De Loof, 1992). For each sample, three biological replicates of ten different sugar or liver fed flies were collected. The tissues were immediately transferred into special tubes containing MagNa Lyser Green Beads (Roche) and frozen in liquid nitrogen. Afterwards all collected samples were homogenized using the MagNa Lyzer (Roche). The total RNA was isolated from the tissue homogenates using the RNeasy Lipid Tissue Mini kit (Qiagen) supplemented with the optional procedure of the DNase treatment (RNase-free DNase set, Qiagen) preventing any genomic DNA contamination.

The quality and quantity of the obtained RNA was verified on the NanoDrop (Thermo Fisher Scientific Inc.). $1 \mu \mathrm{g}$ RNA of each sample was reverse transcribed by using the SuperScript III Reverse Transcriptase Kit (Invitrogen) and random hexamer primers as described in the manufacturer's protocol. The obtained cDNA was diluted tenfold before starting the experiment.

\subsection{Molecular cloning of the adipokinetic hormone receptor}

Because the genome of $S$. crassipalpis is unknown and only a short fragment of a putative SarcrAKHR was present in the transcriptome, there was no possibility to design full length gene specific PCR primers. Different PCR strategies were used to obtain the full length sequence. All primers used in this study were produced by Sigma-Aldrich. All obtained products of the correct expected length were purified using the GenElute Gel Extraction Kit (Sigma-Aldrich) and cloned into a pCR4-TOPO TA sequencing vector (Invitrogen). The cloned vectors were transformed into One Shot TOP10 chemically competent Escherichia coli cells (Invitrogen). The cells were grown overnight at $37^{\circ} \mathrm{C}$ on Luria-Bertani (LB) agar plates (35 g/l, Sigma-Aldrich) with ampicillin $(50 \mu \mathrm{g} / \mathrm{ml}$, Invitrogen). Single colonies were transferred into LB liquid medium (25 g/l, Sigma-Aldrich) with ampicillin $(100 \mu \mathrm{g} / \mathrm{ml})$ and grown overnight at $37^{\circ} \mathrm{C}$ in the shaking incubator. The plasmids were isolated using the GenElute HP Plasmid Miniprep Kit (Sigma-Aldrich) and sequenced using M13 primers unless stated otherwise (LGC Genomics, Germany).

We designed primer pairs (Table 1) based on the best conserved regions of flies' AKHR sequences. Three related species with a complete genome available, namely $D$. melanogaster (GenBank Acc. No. NM_205917.3), Glossina morsitans (GenBank Acc. No. HQ640948.1) and Musca domestica (GenBank Acc. No. NM_001309065.1), were aligned using the online tool Clustal Omega provided by EMBLEBI (http://www.ebi.ac.uk/Tools/msa/clustalo/) and analyzed for the best regions for primers design (Suppl. Fig. 1). Each PCR reaction mixture contained $0.5 \mu$ Advantage II polymerase mix (Clontech), $5 \mu \mathrm{l} 10 \times$ Adventage PCR buffer (Clontech), $1 \mu$ dNTP mixture (10 mM each), $1 \mu \mathrm{l}$ forward and reverse primers $(10 \mu \mathrm{M}), 38.5 \mu \mathrm{l}$ MQ and $3 \mu \mathrm{l}$ cDNA of the fat body. The amplification PCR program contained an initial denaturation step of $95{ }^{\circ} \mathrm{C}$ for $180 \mathrm{~s}$ followed by 30 cycles of $94{ }^{\circ} \mathrm{C}$ for $30 \mathrm{~s}$, gradient of $50{ }^{\circ} \mathrm{C}-70^{\circ} \mathrm{C}$ for $60 \mathrm{~s}$, $68{ }^{\circ} \mathrm{C}$ for $60 \mathrm{~s}$ and a final elongation step of $68^{\circ} \mathrm{C}$ for $600 \mathrm{~s}$. The 
Table 1

Primer pairs used to pick up the Sarcophaga crassipalpis AKH receptor encoding cDNA sequence. Amplification took place for the combination of Dromo forward and Musdo reverse primers. All primers were designed based on conserved regions of Drosophila melanogaster (Dromo), Glossina morsitans (Glomo) and Musca domestica (Musdo) AKH receptor cDNA (See also supplementary Fig. 1).

\begin{tabular}{lll}
\hline Name & Forward primer sequence & Reverse primer sequence \\
\hline Drome AKHR & $5^{\prime}$-GCCATCGCCGATCTAAT-3' & $5^{\prime}$-GTCATCGTTGGAACGC-3' \\
Glomo AKHR & $5^{\prime}$-CGCGTATTGATATTATATGTTAATG-3' & $5^{\prime}$-ACATCATCGTTTGAACGAC-3' \\
Musdo AKHR & $5^{\prime}$-TGCATTTGGCAATAGCCG-3' & $5^{\prime}$-ATCATCGTTGGAACGGC-3' \\
\hline
\end{tabular}

results of the PCR reactions were verified using $1 \%$ agarose gel electrophoresis $(100 \mathrm{~V} / 100 \mathrm{~mA}$ for $1 \mathrm{~h})$. The amplification of the product took place in the three lowest annealing temperatures of $50{ }^{\circ} \mathrm{C}, 54^{\circ} \mathrm{C}$ and $58.8^{\circ} \mathrm{C}$ for the combination of a forward primer of $D$. melanogaster AKHR (5'-GCCATCGCCGATCTAAT-3') and a reverse primer of $M$. domestica AKHR (5'-ATCATCGTTGGAACGGC$\left.3^{\prime}\right)$.

To establish the $\mathrm{N}$-terminal sequence, a forward primer was designed based on the short transcriptome sequence of Sarcophaga crassipalpis (Hahn transcriptome: HAHN.FLY.6451.C1) and genomic sequence data of Neobelliera bullata, formerly called Sarcophaga bullata (GenBank Acc. No. JXPI0114051.1).

Rapid Amplification of cDNA Ends (RACE) was performed in order to find the $\mathrm{C}$-terminal sequence of the receptor. The oligodT anchor primer (5'-GACCACGCGTATCGATGTCGACTTTTTTTTTTTT TTTT-3') necessary for RACE was already added at the level of cDNA synthesis (instead of random hexamer primers). The PCR mixture contained $0.5 \mu \mathrm{l}$ Advantage II polymerase mix (Clontech), $5 \mu \mathrm{l}$ PCR buffers, $1 \mu \mathrm{l}$ nucleotides mixture ( $10 \mathrm{mM}$ each), $1 \mu \mathrm{l}$ gene specific (5'-TCATTTGCAGCGAATTTCACA-3') $(12.5 \mu \mathrm{M})$ and anchor primers (5'-GACCACGCGTATCGATGTCGAC-3') $(37.5 \mu \mathrm{M}), 38.5 \mu \mathrm{l}$ MQ and $3 \mu \mathrm{l}$ undiluted fat body cDNA. The used PCR program had an initial denaturation step of $95^{\circ} \mathrm{C}$ for $180 \mathrm{~s}$ followed by 30 cycles of $94{ }^{\circ} \mathrm{C}$ for $45 \mathrm{~s}, 60^{\circ} \mathrm{C}$ for $60 \mathrm{~s}, 68^{\circ} \mathrm{C}$ for $60 \mathrm{~s}$ and a final elongation step of $68^{\circ} \mathrm{C}$ for $600 \mathrm{~s}$.

The full AKHR sequence was amplified by a PCR reaction using a specific forward (5'-CACCATGACAGAGTCCGAGATAA-3') primer with the 'CACC' Kozak sequence added to the $5^{\prime}$ side $(\ldots)$ and reverse (5'-TTATGTTTTTTGTTGTAGATTAG-3') primer. The PCR mixture was composed of $0.5 \mu \mathrm{l}$ Advantage II polymerase mix (Clontech), $5 \mu \mathrm{l} 10 \times$ Advantage PCR buffer (Clonetech), $1 \mu \mathrm{l}$ dNTP mixture $(10 \mathrm{mM}$ each), $1 \mu \mathrm{l}$ forward and reverse primers $(10 \mu \mathrm{M}), 38.5 \mu \mathrm{l} \mathrm{MQ}$ and $3 \mu \mathrm{l}$ cDNA of the fat body. The amplification program consisted of an initial denaturation step of $95^{\circ} \mathrm{C}$ for $180 \mathrm{~s}$, followed by 35 cycles of $94^{\circ} \mathrm{C}$ for $30 \mathrm{~s}, 60^{\circ} \mathrm{C}$ for $60 \mathrm{~s}, 68^{\circ} \mathrm{C}$ for $120 \mathrm{~s}$ and a final elongation step of $68^{\circ} \mathrm{C}$ for $600 \mathrm{~s}$. The analyzed and purified PCR product was subsequently cloned into a pcDNA3.1/V5-His-TOPO TA expression vector (Invitrogen) and sequenced using T7 and BGH primers (LGC Genomics). Bacteria containing the plasmids with the insert of the correct sequence and right orientation were transferred into $150 \mathrm{ml} \mathrm{LB}$ medium with ampicillin $(100 \mu \mathrm{g} / \mathrm{ml}$, Invitrogen $)$ and grown overnight at $37^{\circ} \mathrm{C}$ in a shaking incubator. Subsequently the plasmid was isolated by means of EndoFree Plasmid Maxi Kit (Sigma-Aldrich) and once again the sequence was confirmed.

\subsection{Quantitative tissue distribution analysis of the adipokinetic hormone receptor}

We analyzed the SarcrAKHR transcript levels using quantitative real-time PCR ( $\mathrm{qPCR}$ ). To obtain trustworthy results of the measurement, we analyzed the expression stability of eight commonly used reference genes (elongation factor $1 \alpha$ (GenBank Acc. No. GQ409484.1), heat shock protein 90 (GenBank Acc. No. AF261773.1), heat shock protein 70 (GenBank Acc. No. AF107338.2), glyceraldehyde 3-phosphate dehydrogenase (GenBank Acc. No. EZ598085.1), ribosomal protein S18 (GenBank Acc. No. GQ409208.1), ribosomal protein L32 (Hahn transcriptome: HAHN.FLY.cl.345), tubulin (Hahn transcriptome: EZ599932.1) and actin (Hanh transcriptome: EUA37Q301ATGMZ)) in all examined tissues and conditions. Using qBase+ software v.2.4 based on the geNorm algorithm (Vandesompele et al., 2002) the three most stable genes were selected (elongation factor $1 \alpha$, heat shock protein 90 and glyceraldehyde 3-phosphate dehydrogenase). All primers were designed with the Primer Express software v.2.0 (Applied Biosystems) (Table 2) and produced by Sigma-Aldrich.

Each PCR mixture contained $10 \mu$ l Fast SYBR Green Master Mix (Applied Biosystems), $1 \mu \mathrm{l}$ forward and $1 \mu \mathrm{l}$ reverse primers $(10 \mu \mathrm{M}), 3 \mu \mathrm{l}$ MilliQ water and $5 \mu \mathrm{l}$ cDNA. The PCR reactions were performed in duplicate on a StepOne Plus System (Applied Biosystems) using the following thermal cycling profile: $95^{\circ} \mathrm{C}$ for $10 \mathrm{~min}$, followed by 40 steps of $95{ }^{\circ} \mathrm{C}$ for $3 \mathrm{~s}$ and $60^{\circ} \mathrm{C}$ for $30 \mathrm{~s}$. The relative expression quantities of the genes were calculated using the $\Delta \Delta \mathrm{Ct}$ method (Livak and Schmittgen, 2001). Changes in tissue distribution analysis were validated by $T$-student test using Statistica 12 software.

\subsection{Structural and phylogenetic analysis}

The SarcrAKHR amino acid sequence was aligned with AKHR sequences of $M$. domestica (GenBank Acc. No. XP_005177426.1), D. melanogaster (GenBank Acc. No. NP_995639.1) and G. morsitans (GenBank Acc. No. AEH25943.1) using Clustal Omega (http://www. ebi.ac.uk/Tools/msa/clustalo/). The transmembrane topology was controlled by the online tool provided by TMHMM Server v. 2.0 (http://www.cbs.dtu.dk/services/TMHMM/). The putative $\mathrm{N}$ linked glycosylation sites were predicted with the NetNGlyc 1.0 server (http://www.cbs.dtu.dk/services/NetNGlyc/).

The phylogenetic analysis of flesh fly AKHR was performed using MEGA 6.1 software. Transmembrane region (TM) 1 - TM7 of all sequences ( $\mathrm{N}$ - and C-terminal parts of the receptors sequences were removed) was used for an alignment with ClustalW. This alignment was then used for a Maximum-likelihood analysis using the Jones-Taylor-Thornton model (1000-fold bootstrap resampling). The $D$. melanogaster FMRFamide receptor (GenBank Acc. No. AAF47700) was used to root the tree.

AKHR amino acid sequences used for phylogenetic analysis derived from $M$. domestica, D. melanogaster, G. morsitans, Anopheles gambiae (GenBank Acc. No. ABD60146.1), Aedes aegypti (GenBank Acc. No. CAY77166.1), Apis mellifera (GenBank Acc. No. NP_001035354.1), Nasonia vitripennis (GenBank Acc. No. NP_001161243.1), B. mori (GenBank Acc. No. NP_001037049.1), Manduca sexta (GenBank Acc. No. ACE00761.1), Tribolium castaneum (GenBank Acc. No. NP_001076809.1), Periplaneta americana (GenBank Acc. No. ABB20590.1), Blatella germanica (Gen Bank Acc. No. ADL60118.1), Gryllus bimaculatus (GenBank Acc. No. ADZ17179.1) and Rhodnius prolixus (GenBank Acc. No. AIJ49751.1). The human gonadotropin-releasing hormone receptor (GenBank Acc. No. NP_000397.1) was also included in the analysis. 
Table 2

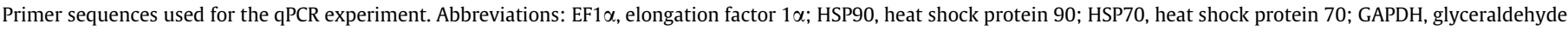
3-phosphate dehydrogenase; RPS18, ribosomal protein S18; RPL32, ribosomal protein L32; TUB, tubulin; ACT, actin; AKHR, adipokinetic hormone receptor.

\begin{tabular}{|c|c|c|}
\hline Gene & Forward primer sequence & Reverse primer sequence \\
\hline EF1 $\alpha$ & 5'-GTCTTCGCCCCCGCTAAC-3' & 5'-AGCTTCGTGGTGCATTTCAAC-3' \\
\hline HSP9O & 5'-GATGCCGACAAGAAAGATAAAGA-3' & 5'-CGGGTCCAGATGGGCTTAGT-3' \\
\hline HSP70 & 5'-CGGGCTAAGCGAACTTTGTCT-3' & 5'-TCCCTCGAAAAGAGCATCGA-3' \\
\hline GAPDH & 5'-TCCTACGATGCCATCAAGGC-3' & 5'-ACGAAATCGGTGGAGACGAC-3' \\
\hline RPS18 & 5'-TATGGCTCTCTCAGTCGCTTCC- ${ }^{\prime}$ & 5'-TGGTGCCCTTCCGTCAATT-3' \\
\hline RPL32 & 5'-AATTGCCCATGGTGTTTCCTC-3' & 5'-TGTGAACGAACACGACCATTG-3' \\
\hline TUB & 5'-CGGAAACTAGCTGATCAATGCA-3' & 5'-ACCACCAAAGGAATGGAACACT-3 \\
\hline ACT & 5'-AACGCAAATACTCCGTCTGGAT-3' & 5'-CGGGACCAGATTCGTCGTA-3' \\
\hline AKHR & 5'-CGTTGCCTGGTGTAGTTCCT-3' & 5'-GGATGCTCCTCCAAGTGGAA-3' \\
\hline
\end{tabular}

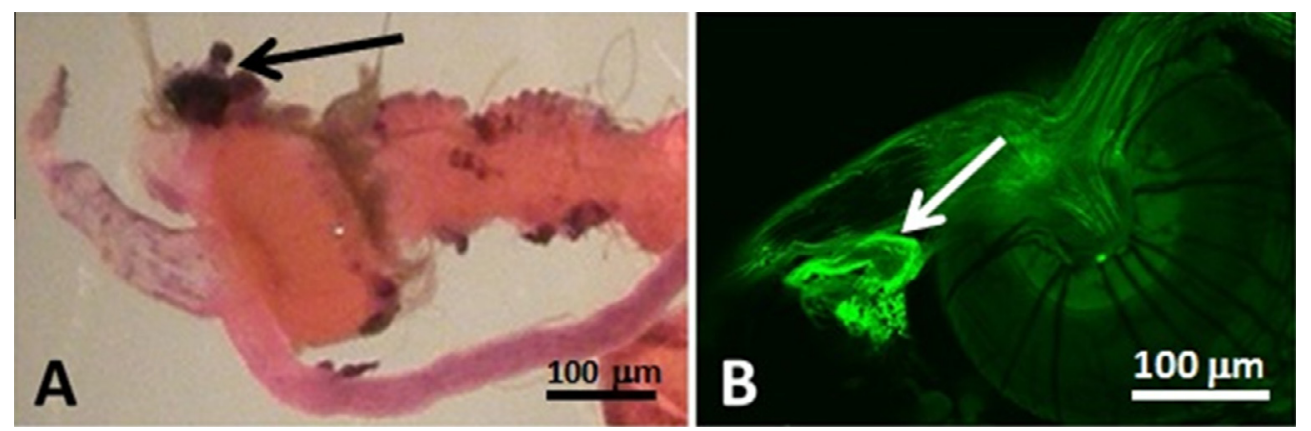

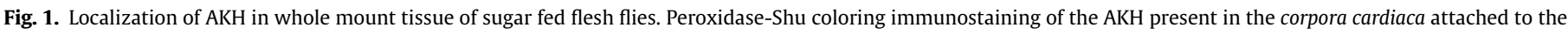

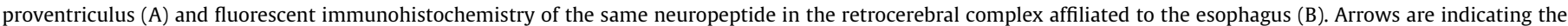
positive signals.

\subsection{Cell culture and transfection}

Chinese hamster ovary (CHO) WTA11 cells that stably express apoaequorin, a zeocin resistance gene, and a promiscuous human $\mathrm{G}_{\alpha 16}$ were cultured in monolayer in Dulbecco's Modified Eagles Medium nutrient mixture F12-Ham (DMEM/F12, Sigma-Aldrich), supplemented with $10 \%$ heat-inactivated fetal calf serum (Invitrogen), $100 \mathrm{IU} / \mathrm{ml}$ penicillin, $100 \mu \mathrm{g} / \mathrm{ml}$ streptomycin (Invitrogen). The cells were cultured in a humid incubator with constant supply of $5 \% \mathrm{CO}_{2}$ at $37^{\circ} \mathrm{C}$.

Cell transfections were performed in T75 flasks at $60-80 \%$ confluency. Transfection medium was prepared using $3 \mathrm{ml}$ DMEM/F12 medium (without additives) containing $45 \mu$ l Lipofectamine LTX (Invitrogen), $7.5 \mu \mathrm{g}$ vector construct and $6 \mu$ l Plus Reagent (Invitrogen) in $5 \mathrm{ml}$ polystyrene tubes. After an incubation period of 30 min at room temperature, the old medium of the cells was removed and the transfection medium was added dropwise to the cells. In addition $3 \mathrm{ml}$ complete medium was added as well. The cells were incubated overnight. An additional $20 \mathrm{ml}$ of the complete cell medium was added for the next overnight incubation prior to the cell screen.

\subsection{Aequorin-bioluminescence assay}

The aequorin-bioluminescence assay was used to measure the calcium release in transfected CHO WTA11 cells upon ligand exposure (as also described in Caers et al., 2016; Verlinden et al., 2015). Transfected cells were detached using 0.2\% EDTA-PBS solution and collected in $10 \mathrm{ml}$ DMEM/F12. Subsequently their number was determined using the NucleoCounter NC-100 (Chemometic). The cells were centrifuged at $800 \mathrm{rpm}$ for $4 \mathrm{~min}$ and the pelleted cells were resuspended to a concentration of $5 \times 10^{6}$ cells $/ \mathrm{ml}$ in DMEM medium containing $0.1 \%$ sterile filtered BSA. Subsequently the whole vial was shielded from the light and $5 \mu \mathrm{M}$ Coelenterazine $\mathrm{H}$ (Invitrogen) was added. The mixture was placed on an orbital shaker at room temperature for $4 \mathrm{~h}$ to allow the coelenterazine $\mathrm{H}$ to enter the cells, which will subsequently lead to reconstitution of the holoenzyme aequorin. $30 \mathrm{~min}$ prior to the screening, the cells were diluted tenfold in 0.1\% BSA/DMEM medium.

Peptide ligands (S. crassipalpis AKH (identical to Phote $\mathrm{HrTH}$ (pQLTFSPDWa)), G. morsitans AKH II (pQLTFSPGWa) and T. castaneum AKH II (pQLNFTPNWa)) (Pepscan, Nederland) were first dissolved in $30 \mu \mathrm{l}$ DMSO (due to their hydrophobic nature) and then diluted in $0.1 \%$ BSA/DMEM medium into the particular concentrations. $50 \mu \mathrm{l}$ of each of the ligand dilutions was distributed in 96wells plate, whereas $50 \mu \mathrm{l}$ of $0.1 \%$ BSA/DMEM medium was used as negative control. $50 \mu \mathrm{l}$ of cell solution was injected by the machine in a well per well manner. The calcium response (the light emission) was measured for $30 \mathrm{~s}$ using a Mithras LB940 (Berthold Technologies). After that time, $50 \mu \mathrm{l}$ of $0.1 \%$ Triton X-100 (Sigma) in $0.1 \% \mathrm{BSA} / \mathrm{DMEM}$ was injected to the cell-ligand mixture and the signal was measured for another $10 \mathrm{~s}$. The cell lysis releases the total remaining cellular calcium content. The ligand specific response was normalized relative to the total signal (ligand and Triton signal, which serves as an indication of the total amount of cells present) using the output file of Microwin2000 (Microtek). Further analysis of three independent duplicate measurements was done in Excel 2010 (Microsoft) and GraphPad Prism 6.

\section{Results}

\subsection{Adipokinetic hormone immunostaining}

A specific antibody staining clearly proved the presence of the AKH peptide in the corpora cardiaca of S. crassipalpis that is situated between esophagus and proventriculus (Fig. 1). AKH seemed to be aggregated in big storage parts of this neurohemal organ.

\subsection{Identification and sequence analysis of adipokinetic hormone receptor}

The PCR amplification reaction resulted in only one cDNA fragment encoding AKHR. The open reading frame including the stop 
Drome

Glomo

Musdo

Sarcr

Drome

Glomo

Musdo

Sarcr

Drome

Glomo

Musdo

Sarcr

Drome

Glomo

Musdo

Sarcr

Drome

Glomo

Musdo

Sarcr

Drome

Glomo

Musdo

Sarcr

Drome

Glomo

Musdo

Sarcr

Drome

Glomo

Musdo

Sarcr
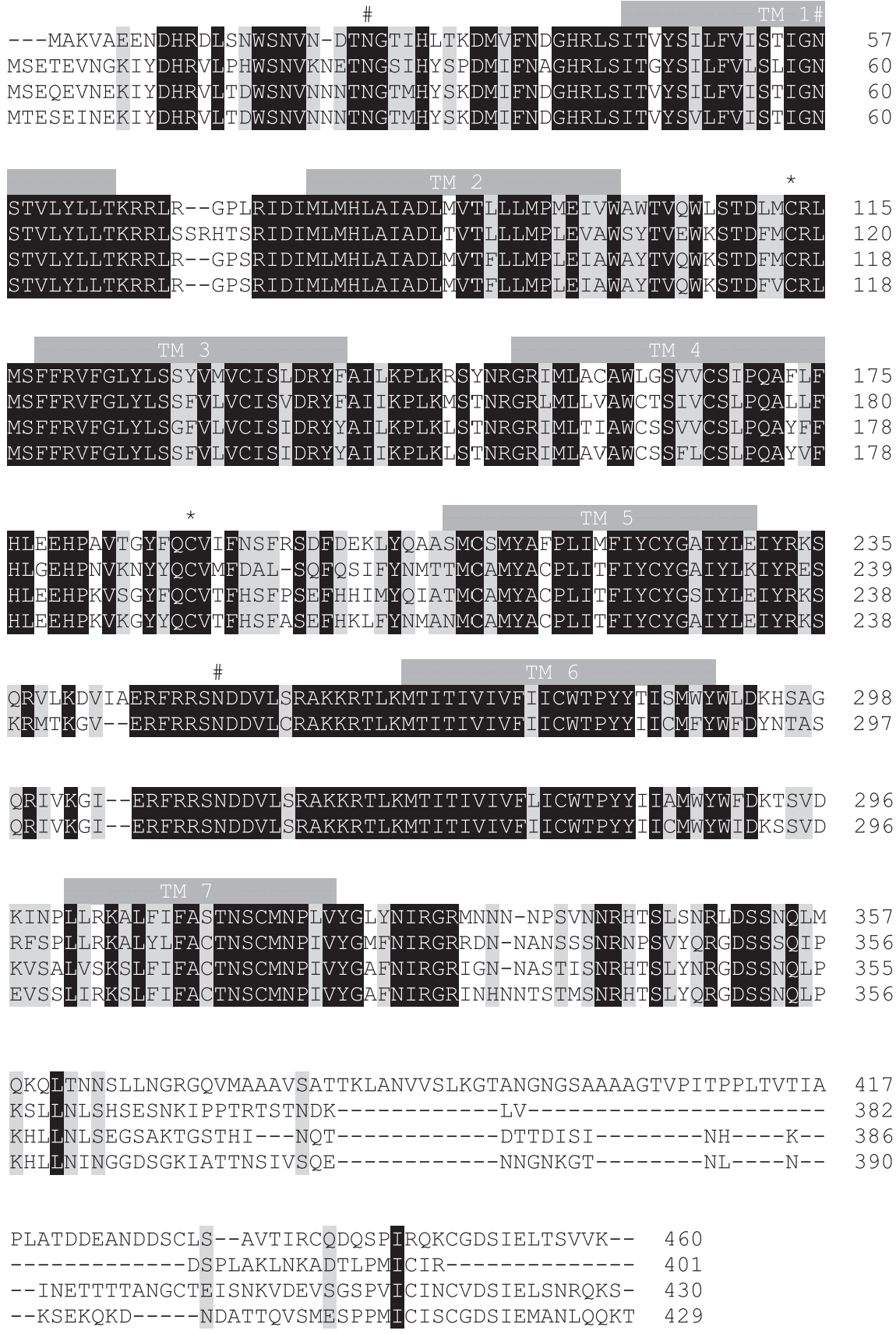

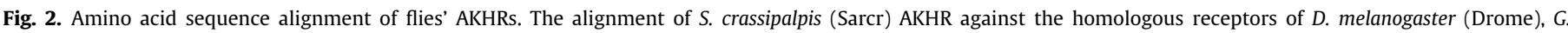

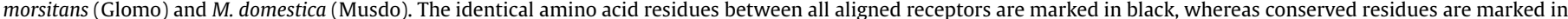

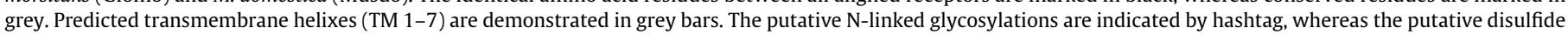
places are indicated by asterisk.

codon contained 1290 nucleotides (Suppl. Fig. 2) which resulted in 429 amino acids residues (Fig. 2). The calculated molecular weight was $49.363 \mathrm{kDa}$.

Transmembrane topology analysis confirms that the AKHR is a GPCR. The typical DRY motif (at the end of the 3rd transmembrane domain) and the LXXXNSXXNPXXY motif (in the 7th transmembrane helix) characteristic for all rhodopsin-like GPCR are present. A possible disulfide bridge can be formed between two cysteine residues (C118-C194) localized on the second and third extracellular loops. Putative N-linked glycosylation could be present on N27, N60 and N333 residues.

\subsection{Phylogenetic analysis}

Phylogenetic analysis (Fig. 3) clearly shows that all flies' AKHR sequences cluster together. Similar to the BLAST search, the closest relative to $S$. crassipalpis AKHR is the receptor sequence of $M$. domestica. Although the sequences of the AKHRs of the major insect orders (Hymenoptera, Coleoptera, Orthoptera, Lepidoptera, Hemiptera) form separate clusters, the phylogenetic relationships between the different groups is not always clear. This is also reflected in some of the rather low bootstrap values. 


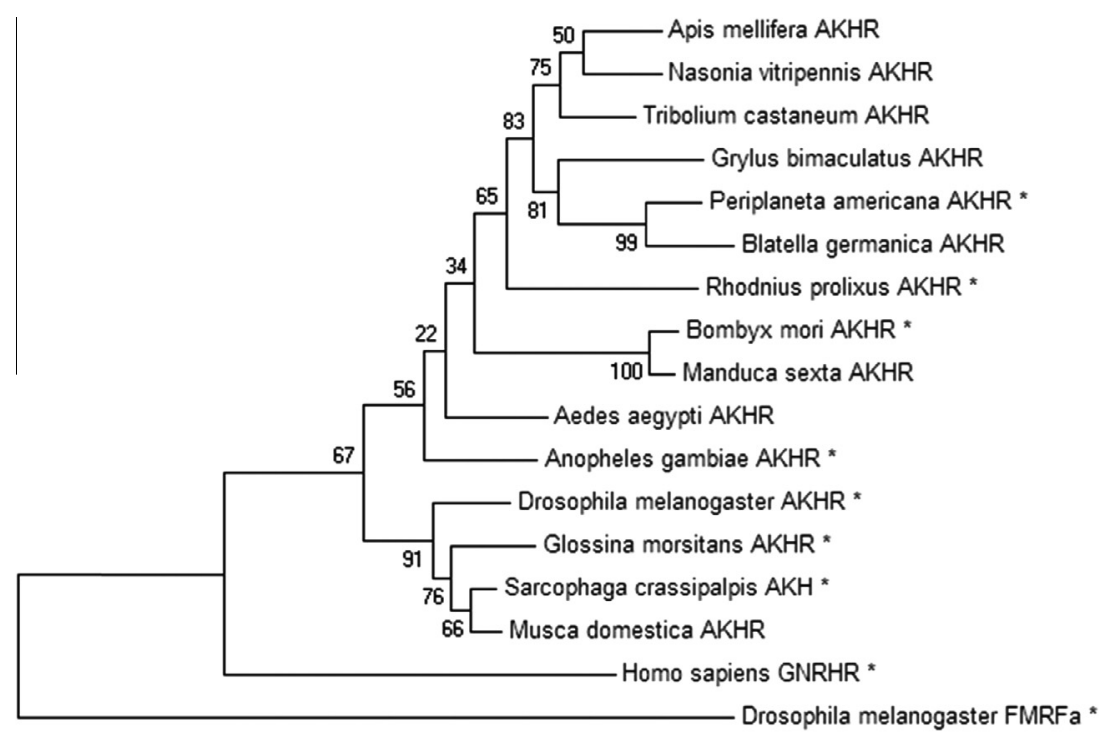

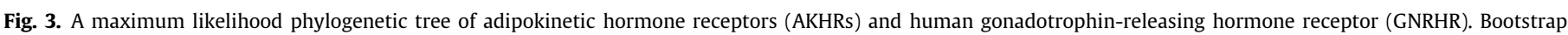

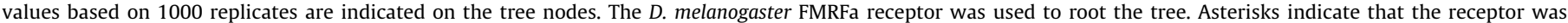
pharmacologically characterized.

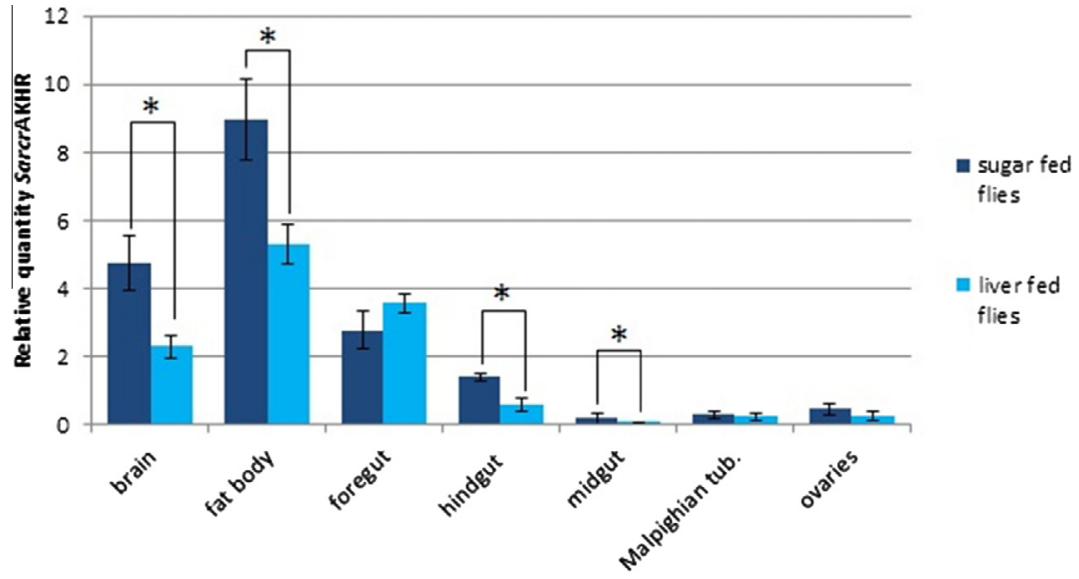

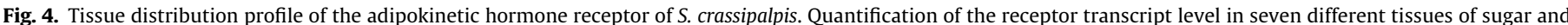

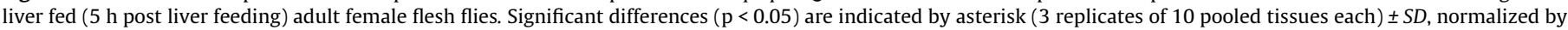
using the geometric mean of elongation factor $1 \alpha$, heat shock protein 90 and glyceraldehyde 3-phosphate dehydrogenase transcript levels.

\subsection{Receptor transcript level tissue distribution analysis}

Based on differences in quantity of AKH peptide observed between sugar and liver fed female flies (Bil et al., 2014), the receptor transcript tissue distribution analysis was also performed on sugar and protein fed ( $5 \mathrm{~h}$ post protein feeding) flies. Significantly reduced AKHR transcript levels were measured in the brain, fat body, hindgut and midgut ( $\mathrm{p}$-values T-student test: $\mathrm{p}_{\text {brain }}=0.020$; $\mathrm{p}_{\mathrm{fat}}$ body $=0.018 ; \quad \mathrm{p}_{\text {hindgut }}=0.018 ; \quad \mathrm{p}_{\text {midgut }}=0.009$ ) of liver fed females (Fig. 4).

In general, AKHR gene expression was the most prominent in the fat body (in sugar as well as in liver fed condition). AKHR transcripts were also highly abundant in the brain, foregut and hindgut.

\subsection{Functional activation and dose response analysis of S. crassipalpis adipokinetic hormone receptor}

S. crassipalpis AKHR was expressed in CHO-WTA11 cells containing a promiscuous $G_{\alpha 16}$ subunit. The receptor dose response activation was tested using three AKH peptides, derived from Dip- tera (S. crassipalpis, G. morsitans) and Coleoptera (T. castaneum) (Fig. 5). Both dipteran AKH peptides, which differ in one amino acid localized on the seventh position (aspartic acid and glycine respectively), gave very similar results and clearly elicited a dosedependent response with $\mathrm{EC}_{50}$ values of 20.82 and $44.91 \mathrm{nM}$ respectively. Maximal receptor activation was achieved with concentration of ten micromolar or higher. No response could be measured for $0.1 \mathrm{nM}$ solutions for both flies' AKHs. TricaAKH II could also activate the receptor although much higher concentrations were needed $\left(\mathrm{EC}_{50}\right.$ value could not be determined since no plateau was reached with the used peptide dilutions. Higher ligand concentrations could not be dissolved properly in low, non-harmful for the cells, percentage of DMSO).

No AKH response was observed for CHO-WTA11 cells transfected with empty pcDNA3.1/V5-His-TOPO TA expression vector construct.

\section{Discussion}

The immunohistochemical staining confirms the large scale abundance of the AKH peptide in the neurohemal organ, corpora 


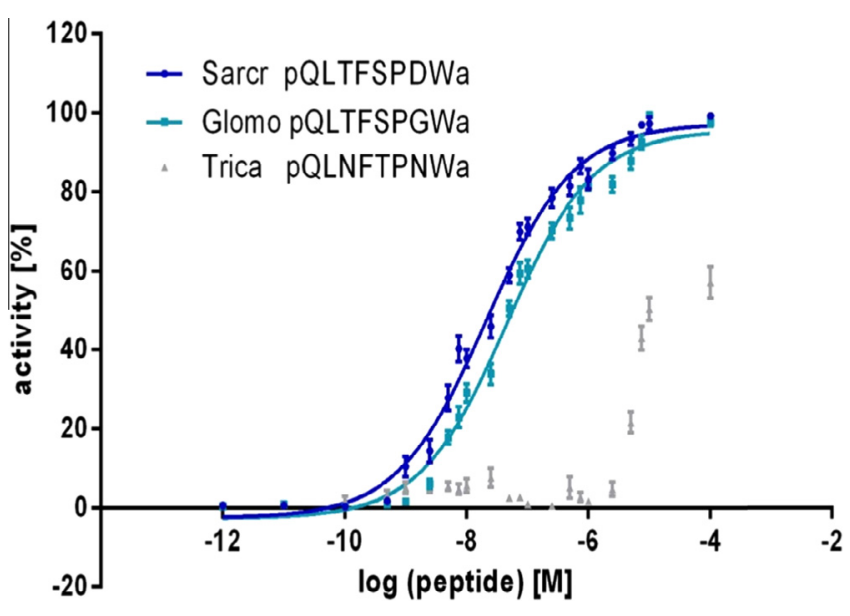

Fig. 5. Dose response curve for bioluminescence responses induced by $S$. crassipalpis AKH (Sarcr), G. morsitans AKH II (Glomo) and T. castaneum AKH II (Trica) in CHO-WTA11 cells transfected with $S$. crassipalpis AKHR (three independent duplicate measurements) \pm SEM.

cardiaca, as was already described in the literature for many other insects (Gäde and Auerswald, 2003; Abdel-Latief and Hoffmann, 2007; Diederen et al., 2002; Rahman et al., 2013).

Sarcophaga crassipalpis

AKHR is a glycoprotein hormone receptor containing seven transmembrane helices characteristic for the family of GPCRs. It also includes specific amino acid motifs typical for the rhodopsin-like family of GPCRs (Costanzi, 2012). AKHRs and the vertebrate gonadotropin-releasing hormone receptors are structurally and evolutionary related (Hauser et al., 1998; Lindemans et al., 2011).

The Diptera order consists of flies and mosquitoes forming two groups derived from a common ancestor (http://www.livescience.com/48663-insect-family-tree-evolution.html). Surprisingly, not all dipteran AKHRs cluster together in our phylogenetic tree, which is similar to the phylogenetic analysis of Attardo et al. (2012). The members of the main orders in our analysis do cluster accordingly, but also the relative position of the different orders is not in agreement with the insect family tree. First of all more sequences from different orders may shed a better light on this, but it may also be that convergent evolution between different insect orders for this specific gene has occurred due to e.g. similar energy needs caused by similar environments.

The highest AKHR transcript levels in S. crassipalpis were measured in the fat body. This corresponds with the main and also best known function of $\mathrm{AKH}$, lipid and carbohydrate mobilization from the fat body during high energetic processes such as flying (Gäde et al., 1997). Also the brain contains high AKHR transcript levels, which is similar as observed in the silkworm, B. mori (Shi et al., 2011). As was also observed in the cockroach $P$. americana high AKHR transcript levels were detected in the initial part of the digestion tract (Bodláková et al., 2016). These authors demonstrated that AKH was indeed involved in the regulation of digestive enzymes. Similarly, AKH was demonstrated to be involved in moving carbohydrates up into the midgut for digestion in the anautogenous black blow fly Phormia regina (Stoffolano et al., 2014), which also points out the presence of the AKHR in the foregut of this fly.

The decrease of the AKHR transcript levels upon a protein meal is not only observed in liver fed S. crassipalpis, but also in blood fed A. gambiae (Kaufmann and Brown, 2006). It can be related to the feeding and digestion processes that provide a lot of different energy carrying molecules passing into the hemolymph which should be consumed first. The lower abundance of AKHR prevents the use of stored reserves and preserves them for future activities or for the 'emergency' case when no food is available. Knockdown of the AKHR in the cricket, G. bimaculatus, resulted in decreased levels of energy carrying molecules in the hemolymph and increased levels of triacylglycerol in the fat body. As expected, this also led to an increase of the feeding frequency of that insect (Konuma et al., 2012). Furthermore, post liver feeding decrease of the AKHR gene expression level corresponds well with the decrease of the AKH peptide observed in liver fed flesh flies (Bil et al., 2014).

The pharmacological results of the SarcrAKHR correspond well with other pharmacological studies of AKHRs. The one amino acid difference (aspartate-glycine difference at the seventh residue) between the two dipteran AKH peptides does not seem to make any difference for receptor activation as observed in $D$. melanogaster, where the synthetic ligand having the alanine-substitution on this position elicits almost the same receptor activation response as observed for the original peptide ligand (Caers et al., 2012). In both flies' ligands the entire essential N-terminal pentapeptide (Gäde and Hayes, 1995) remains identical which guarantees high binding affinity. Coleopteran T. castaneum AKH II peptide differs much more in its sequence compared to the previous ones. This is also reflected in its receptor activation efficiency. TricaAKH II has an amino acid substitution on the 3rd position (threonine into asparagine) and also on the 5th position (serine into threonine) additionally to an amino acid substitution on the 7th position (aspartate or glycine to asparagine). These residues seemingly play an important role in ligand-receptor binding and activation as demonstrated in D. melanogaster and A. gambiae (Caers et al., 2012). Nevertheless, both substitutions (3th and 5th position) seen in TricaAKH II represent changes of one amino acid with the polar uncharged side chain for the other one with the same properties. They still allow this peptide to bind and activate the receptor although much higher concentrations are needed. Substitutions by amino acids with different nature are described to almost completely abolish interaction with AKH receptors (Caers et al., 2012, 2016).

\section{Acknowledgments}

The authors thank Professor Jan Veenstra for his kind gift of AKH antibodies, as well as laboratory technician Ria Vanlaer for immunohistochemistry staining. We would like to thank Marc Parmentier (Free University of Brussels, Belgium) and Michel Detheux (Euroscreen S.A., Belgium) for providing CHO-WTA11 as well as Dr. Sven Zels, Charline Borghgraef and Esra Baytemur for their support in cell culture experiments. This research was supported by FWOFlanders (Project G.0405.09N10), the KU Leuven Research Foundation (GOA/11/002). M.B. was supported by FLOF (faculty research fund) and H.V. was supported by FWO.

\section{Appendix A. Supplementary data}

Supplementary data associated with this article can be found, in the online version, at http://dx.doi.org/10.1016/j.jinsphys.2016.04. 001.

\section{References}

Abdel-Latief, M. Hoffmann, K.H. 2007. The adipokinetic hormones in the fall armyworm, Spodoptera frugiperda: cDNA cloning, quantitative real time RT-PCR analysis, and gene specific localization. Insect Biochem. Mol. Biol. 37, 999-1014. Attardo, G.M., Higgs, S., Klingler, K.A., Vanlandingham, D.L., Raikhel, A.S., 2003. RNA interference-mediated knockdown of a GATA factor reveals a link to anautogeny in the mosquito Aedes aegypti. Proc. Natl. Acad. Sci. U.S.A. 100, 13374-13379. 
Attardo, G.M., Benoit, J.B., Michalkova, V., Yang, G., Roller, L., Bohova, J., Takac, P., Aksoy, S., 2012. Analysis of lipolysis underlying lactation in the tsetse fly, Glossina morsitans. Insect Biochem. Mol. Biol. 42, 360-370.

Bil, M., Broeckx, V., Landuyt, B., Huybrechts, R., 2014. Differential peptidomics highlights adipokinetic hormone as key player in regulating digestion in anautogenous flesh fly, Sarcophaga crassipalpis. Gen. Comp. Endocrinol. 208, 4956.

Bodláková, K., Jedlička, P., Kodrik, D., 2016. Adipokinetic hormones control amylase activity in the cockroach (Periplaneta americana) gut. Insect Sci.

Caers, J., Janssen, T., Van Rompay, L., Broeckx, V., Van Den Abbeele, J., Gäde, G., Schoofs, L., Beets, I., 2016. Characterization and pharmacological analysis of two adipokinetic hormone receptor variants of the tsetse fly, Glossina morsitans morsitans. Insect Biochem. Mol. Biol. 70, 73-84.

Caers, J., Peeters, L., Janssen, T., De Haes, W., Gäde, G., Schoofs, L., 2012. Structureactivity studies of Drosophila adipokinetic hormone (AKH) by a cellular expression system of dipteran AKH receptors. Gen. Comp. Endocrinol. 177, 332-337.

Costanzi, S., 2012. Homology modeling of class a G protein-coupled receptors Methods Mol. Biol. 857, 259-279.

Diederen, J.H.B., Oudejans, R.C.H.M., Harthoorn, L.F., Van Der Horst, D.J., 2002. Cell biology of the adipokinetic hormone-producing neurosecretory cells in the locust corpus cardiacum. Microsc. Res. Tech. 56, 227-236.

Gäde, G., 2009. Peptides of the adipokinetic hormone/red pigment-concentrating hormone family a new take on biodiversity. Trends Comp. Endocrinol. Neurobiol. 1163, 125-136.

Gäde, G., Auerswald, L., 2003. Mode of action of neuropeptides from the adipokinetic hormone family. Gen. Comp. Endocrinol. 132, 10-20.

Gäde, G., Hayes, T.K., 1995. Structure-activity relationships for Periplaneto americana hypertrehalosemic hormone. I: the importance of side chains and termini. Peptides 16, 1173-1180.

Gäde, G., Hoffmann, K.H., Spring, J.H., 1997. Hormonal regulation in insects: facts, gaps, and future directions. Physiol Rev. 77, 963-1032.

Goldsworthy, G.J., Jutsum, A.R., Robinson, N.L., 1979. Substrate utilization and flight speed during tethered flight in the locust. J. Insect Physiol. 25, 183-185.

Goldsworthy, G.J., Opoku-Ware, K., Mullen, L.M., 2005. Adipokinetic hormone and the immune responses of locusts to infection. Trends Comp. Endocrinol. Neurobiol. 1040, 106-113.

Hauser, F., Sondergaard, L., Grimmelikhuijzen, C.J.P., 1998. Molecular cloning, genomic organization and developmental regulation of a novel receptor from Drosophila melanogaster structurally related to gonadotropin-releasing hormone receptors from vertebrates. Biochem. Biophys. Res. Commun. 249, $822-828$.

Isabel, G., Martin, J.R., Chidami, S., Veenstra, J.A., Rosay, P., 2005. AKH-producing neuroendocrine cell ablation decreases trehalose and induces behavioral changes in Drosophila. Am. J. Physiol. Regul. Integr. Comp. Physiol. 288, R531R538.

Kaufmann, C., Brown, M.R., 2006. Adipokinetic hormones in the African malaria mosquito, Anopheles gambiae: Identification and expression of genes for two peptides and a putative receptor. Insect Biochem. Mol. Biol. 36, 466-481.

Kodrik, D., 2008. Adipokinetic hormone functions that are not associated with insect flight. Physiol. Entomol. 33, 171-180.

Kodrik, D., Socha, R., Simek, P., Zemek, R., Goldsworthy, G.J., 2000. A new member of the $\mathrm{AKH} / \mathrm{RPCH}$ family that stimulates locomotory activity in the firebug, Pyrrhocoris apterus (Heteroptera). Insect Biochem. Mol. Biol. 30, 489-498.
Kodrik, D., Socha, R., Syrova, Z., Zemek, R., 2005. The effect of constant darkness on the content of adipokinetic hormone, adipokinetic response and walking activity in macropterous females of Pyrrhocoris apterus (L.). Physiol. Entomol. 30, 248-255.

Kodrik, D., Vinokurov, K., Tomčala, A., Socha, R., 2012. The effect of adipokinetic hormone on midgut characteristics in Pyrrhocoris apterus L. (Heteroptera). J. Insect Physiol. 58, 194-204.

Konuma, T., Morooka, N., Nagasawa, H., Nagata, S., 2012. Knockdown of the adipokinetic hormone receptor increases feeding frequency in the two-spotted cricket Gryllus bimaculatus. Endocrinology 153, 3111-3122.

Lindemans, M., Janssen, T., Beets, I., Temmerman, L., Meelkop, E., Schoofs, L., 2011. Gonadotropin-releasing hormone and adipokinetic hormone signaling systems share a common evolutionary origin. Front. Endocrinol. (Lausanne) 2, 16.

Livak, K.J., Schmittgen, T.D., 2001. Analysis of relative gene expression data using real-time quantitative PCR and the 2(T)(-Delta Delta C) method. Methods 25 402-408.

Marco, H.G., Simek, P., Gäde, G., 2011. The first decapeptide adipokinetic hormone $(\mathrm{AKH})$ in Heteroptera: a novel AKH from a South African saucer bug, Laccocoris spurcus (Naucoridae, Laccocorinae). Peptides 32, 454-460.

Meulemans, W., De Loof, A., 1992. Transport of the cationic fluorochrome rhodamine 123 in an insect's Malpighian tubule: indications of a reabsorptive function of the secondary cell type. J. Cell Sci. 101 (Pt 2), 349-361.

Rahman, M.M., Neupert, S., Predel, R., 2013. Neuropeptidomics of the Australian sheep blowfly Lucilia cuprina (Wiedemann) and related Diptera. Peptides 41, 31 37.

Scarborough, R.M., Jamieson, G.C., Kalish, F., Kramer, S.J., Mcenroe, G.A., Miller, C.A., Schooley, D.A., 1984. Isolation and primary structure of 2 peptides with cardioacceleratory and hyperglycemic activity from the corpora cardiaca of Periplaneta-americana. Proc. Natl. Acad. Sci. U.S. A. Biol. Sci. 81, 5575-5579.

Shi, Y., Huang, H.S., Deng, X.Y., He, X.B., Yang, J.W., Yang, H.P., Shi, L.E., Mei, L.J., Gao, J.M., Zhou, N.M., 2011. Identification and functional characterization of two orphan g-protein-coupled receptors for adipokinetic hormones from silkworm Bombyx mori. J. Biol. Chem. 286, 42390-42402.

Staubli, F., Jorgensen, T.J.D., Cazzamali, G., Williamson, M., Lenz, C., Sondergaard, L., Roepstorff, P., Grimmelikhuijzen, C.J.P., 2002. Molecular identification of the insect adipokinetic hormone receptors. Proc. Natl. Acad. Sci. U.S.A. 99, 34463451.

Stoffolano Jr., J.G., Croke, K., Chambers, J., Gäde, G., Solari, P., Liscia, A., 2014. Role of Phote-HrTH (Phormia terraenovae hypertrehalosemic hormone) in modulating the supercontractile muscles of the crop of adult Phormia regina Meigen. J. Insect Physiol. 71, 147-155.

Stone, J.V., Mordue, W., Batley, K.E., Morris, H.R., 1976. Structure of locust adipokinetic hormone, a neurohormone that regulates lipid utilization during flight. Nature 263, 207-211.

Van der Horst, D.J., 2003. Insect adipokinetic hormones: release and integration of flight energy metabolism. Comp. Biochem. Physiol. B Biochem. Mol. Biol. 136, 217-226.

Vandesompele, J., De Preter, K., Pattyn, F., Poppe, B., Van Roy, N., De Paepe, A., Speleman, F., 2002. Accurate normalization of real-time quantitative RT-PCR data by geometric averaging of multiple internal control genes. Genome Biol. 3.

Verlinden, H., Vleugels, R., Verdonck, R., Urlacher, E., Broeck, J.V., Mercer, A., 2015. Pharmacological and signalling properties of a D2-like dopamine receptor (Dop3) in Tribolium castaneum. Insect Biochem. Mol. Biol. 56, 9-20. 